\title{
Implantation of bone mesenchymal stem cells overexpressing miRNA-705 mitigated ischemic brain injury
}

\author{
MENG JI, WEI WANG, SHUJUAN LI and WENLI HU \\ Department of Neurology, Beijing Chao-yang Hospital, Affiliate of Capital Medical University, Beijing 100020, P.R. China
}

Received July 24, 2016; Accepted August 8, 2017

DOI: $10.3892 / \mathrm{mmr} .2017 .7626$

\begin{abstract}
Ischemic brain damage remains the major cause of death and disability worldwide. Bone mesenchymal stem cell (BMSC) transplantation has been identified to serve important roles in cerebral infarction due to its multi-directional differentiation and proliferative ability. However, the function of miR-705 combined with BMSCs on ischemic brain injury remains to be fully elucidated. In the present study, an ischemic brain injury mouse model was constructed, and the mice were injected with BMSCs infected by lentiviral particles expressing miR-705 (BMSCs-Ad-miR-705) to explore the mechanism by which BMSCs-Ad-miR-705 mitigates neurological deficits in ischemic brain damage. In the sham group, no significant neurological injury evaluated via neurological deficit scores was identified, the morphological structure of brain stained with HE was almost normal, and few apoptotic cells were detected by TUNEL assay. However, the PBS group exhibited significant brain damage $(\mathrm{P}<0.05)$. BMSCs-Ad (BMSCs infected with control lentiviral particles) and BMSCs-Ad-miR-705 markedly mitigated neurological injury, suppressed morphological damage and inhibited neuronal apoptosis, however promoted the mRNA levels of brain-derived neurotrophic factor (BDNF) and vascular endothelial growth factor (VEGF) examined by reverse transcription-quantitative polymerase chain reaction and western blotting. Notably, BMSCs-Ad-miR-705 improved the outcome of BMSCs-Ad transplantation. These data indicated that BMSCs-Ad-miR-705 promoted the secretion of
\end{abstract}

Correspondence to: Dr Wenli Hu, Department of Neurology, Beijing Chao-yang Hospital, Affiliate of Capital Medical University, 8 Gongren Tiyuchang Nanlu, Chaoyang, Beijing 100020, P.R. China E-mail:wenlihu2016@163.com

Abbreviations: BDNF, brain-derived neurotrophic factor; ECA, external carotid artery; HE, hematoxylin eosin; ICA, internal carotid artery; MCAO, middle cerebral artery occlusion; miR-705, miRNA-705; NDs, neurological deficit scores; RT-qPCR, reverse transcription-quantitative polymerase chain reaction; VEGF, vascular endothelial growth factor

Key words: bone mesenchymal stem cells, brain-derived neurotrophic factor, ischemic brain injury, miRNA-705, vascular endothelial growth factor
VEGF and BDNF, suppressed neuronal apoptosis, and stimulated neuronal regeneration, in turn mitigating the impairment of ischemic brain damage.

\section{Introduction}

Despite the development of medical technology, cerebrovascular disease, approximately $80 \%$ of which is ischemic brain damage, remains as the major cause of death and disability worldwide (1). Based on its multi-directional differentiation and proliferation ability, bone mesenchymal stem cell (BMSC) transplantation has attracted increasing attention (2). When BMSCs were injected into mice with a cerebral infarction, the cells were observed to survive in the ischemic brain, migrate to the cerebral infarct and promote neurological recovery (3). In addition, BMSCs have been observed to secrete various cytokines and neurotrophic factors, such as brain-derived neurotrophic factor (BDNF) and vascular endothelial growth factor (VEGF) (4). BDNF has been demonstrated to promote functional recovery of the ischemia brain (5), and VEGF induces neuroprotection and neurogenesis following cerebral ischemia (6).

MicroRNAs (miRNAs) are a class of single-strand non-coding RNAs consisting of 18-24 nucleotides in length (7), which are involved in multiple physiological and pathological processes (8) by negatively regulating gene expression at the post-transcriptional level by either target mRNA degradation or translational repression (9). Increasing evidence has demonstrated that miRNAs are crucial for BMSC differentiation (10). microRNA (miR)-705 was first reported to be associated with steatohepatitis (11). Then miR-705 was identified to be a novel negative regulator of cell lineage commitment of BMSCs by directly targeting the homeobox A10 (HOXA10) mRNA 3' untranslated region (12). Furthermore, the transfection of miR-705 mimics or inhibitors in BMSCs affected the expression of forkhead box O1 (FoxO1) (13), suggesting miR-705-transfected BMSCs might be a good model for study of function. The present study indicated that the expression of miR-705 was associated with ischemic brain damage of mice. Although previous studies have observed that BMSCs could rescue ischemic brain damage (2-4), the role of miR-705 combined with BMSCs in ischemic brain injury remained to be elucidated.

In the present study, a lentiviral expression vector was designed for miR-705 and BMSCs were infected with lentiviral 
particles. Subsequently, the BMSCs overexpressing miR-705 were injected into mice with ischemic brain injuries induced by middle cerebral artery occlusion (MCAO). The effect of BMSCs infected with miR-705 on neurological deficit scores, neuronal morphology and cell apoptosis was investigated in order to confirm the function of miR-705 in ischemic brain damage therapy.

\section{Materials and methods}

Experimental animals. A total of 50 male C57BL/6J mice in a SPF grade, aged 8 weeks, weight $200+10$ g, were purchased from Beijing Huafukang Biotechnology Company (Beijing, China). Mice were housed in a colony room under controlled temperature $\left(22+3^{\circ} \mathrm{C}\right)$, and a 12:12 light-dark cycle, with food and water available. All animal care and use was approved by the ethics committee of Beijing Chao-yang Hospital.

Preparation of BMSCs. The C57BL/6J male mice in SPF grade were anesthetized with chloral hydrate $(30 \mathrm{mg} / 100 \mathrm{~g}$; Sigma-Aldrich; Merck KGaA, Darmstadt, Germany) via peritoneal injection. The diaphyses of the bones were cut in sterile conditions in order to open the marrow cavity. Bone marrow was pushed out of the bone with a syringe with a no. 9 needle containing Dulbecco's modified Eagle's medium (DMEM; Gibco; Thermo Fisher Scientific, Inc., Waltham, MA, USA) containing 10\% fetal bovine serum (Gibco; Thermo Fisher Scientific, Inc.). A total of $5 \mathrm{ml}$ bone marrow was aspired and then mixed with phosphate-buffered saline (PBS) at a volume ratio of $1: 1$. The mixture was added to the lymphocyte separation solution at a volume ratio of $2: 1$, and centrifuged at $3,500 \mathrm{x} \mathrm{g}$ for $20 \mathrm{~min}$ at $4^{\circ} \mathrm{C}$. The cloudy layer (lymphocytes) was harvested and washed in serum-free DMEM twice after centrifugation at $1,500 \mathrm{x}$ g for $10 \mathrm{~min}$ at $4^{\circ} \mathrm{C}$. The cells were incubated in complete medium in a humidified environment with $5 \% \mathrm{CO}_{2}$ at $37^{\circ} \mathrm{C}$. The medium was first changed after $24 \mathrm{~h}$, and changed again after $48 \mathrm{~h}$ to remove non-adherent cells. Cells were diluted with $9 \mathrm{ml}$ Hanks buffer and mixed with $1 \mathrm{ml} 0.4 \%$ trypan blue for detection of the cell viability. The BMSC passage was completed at a ratio of 1:2. The cells of passage 3 were used in the following experiments.

Vector construction of a lentiviral expression vector for miR-705. The target sequence of miR-705 was amplified by polymerase chain reaction (PCR) and was inserted into the pCDH-CMV-MCS-EF1-cop-green fluorescent protein (GFP) plasmid (System Biosciences, Mountain View, CA). The recombinant plasmid was verified by restriction endonuclease analysis and DNA sequencing. 293T cells were purchased from American Type Culture Collection and cultured in $25 \mathrm{~cm}^{2}$ flask containing $4 \mathrm{ml}$ DMEM with $10 \%$ fetal bovine serum (Gibco; Thermo Fisher Scientific, Inc.). At a density of $70-90 \%, 293 \mathrm{~T}$ cells were cotransfected with lentiviral vector pCDH-CMV-MCS-EF1-copGFP-miR-705 (System Biosciences) and lentivirus package plasmid mixture. After $8 \mathrm{~h}$, the old medium was replaced with fresh medium for $48 \mathrm{~h}$ incubation at $37^{\circ} \mathrm{C}$. The supernatant was collected and filtered with a $0.45 \mu \mathrm{m}$ membrane. The control cells were transfected with the control lentiviral vector. Virus titers were measured according to the expression of GFP. Furthermore, the expression level of miR-705 was measured by reverse transcription-quantitative PCR (RT-qPCR). Lentiviral particles were collected and stored at $-80^{\circ} \mathrm{C}$ for further experiments.

BMSCs were seeded into the $10 \mathrm{~cm}$ plate. At a density of $80 \%$, cells were infected with lentiviral particles of miR-705 [BMSCs-adenovirus (Ad)-miR-705], and the control was incubated with control lentiviral particles (BMSCs-Ad). The cell density was adjusted to $1 \times 10^{6}$ cells/ml prior to transplantation.

Animal model and grouping. Mice were fixed on the table, anesthetized with an intraperitoneal injection of chloral hydrate $(30 \mathrm{mg} / 100 \mathrm{~g})$, and subjected to MCAO for ischemic brain damage. In brief, the skin of the middle of the neck was opened, and the left common carotid artery, external carotid artery (ECA) and internal carotid artery (ICA) were exposed. The origin of MCA was occluded with a monofilament suture with a distal cylinder from ECA to ICA. The sham-operative mice received the same surgery, except that the filament was inserted only $10 \mathrm{~mm}$ and withdrawn a minute later.

A total of 40 mice underwent $24 \mathrm{~h}$ reperfusion after $2 \mathrm{~h}$ ischemic brain injury and were divided into four groups as follows: i) Sham group, underwent sham operation and received PBS ( $n=10)$; ii) PBS group, subjected to MCAO and received PBS $(n=10)$; iii) BMSCs-Ad group, subjected to MCAO and injected with BMSCs-Ad $\left(200 \mu 1,1 \times 10^{6}\right.$ cells $\left./ \mathrm{ml}\right)$ through ECA $(n=10)$; iv) BMSCs-Ad-miR-705 group, subjected to MCAO and injected with BMSCs infected with Ad-miR-705 $\left(200 \mu 1,1 \times 10^{6}\right.$ cells/ml) via ECA $(\mathrm{n}=10)$. After 7 days, brain tissues were isolated for further experiments.

$R T-q P C R$. Total RNA was extracted from the brain tissues using TRIzol reagent (Invitrogen; Thermo Fisher Scientific, Inc.) according to the manufacturer's instructions. The purity of RNA was determined by absorbance at $260 \mathrm{~nm}$ and $280 \mathrm{~nm}$, and RNA integrity was verified by agarose gel electrophoresis. A total of $5 \mathrm{mg}$ of RNA was converted to cDNA using the iScript cDNA Synthesis kit (Bio-Rad Laboratories, Inc., Hercules, CA, USA). RT-qPCR was performed using Ultra SYBR mixture (CW Biotech, Beijing, China) containing SYBR Green I staining on a CFX96 ${ }^{\mathrm{TM}}$ Real-time system (Bio-Rad Laboratories, Inc., Hercules, CA, USA). The temperature protocol for PCR was as follows: $95^{\circ} \mathrm{C}$ for $5 \mathrm{~min}$, $95^{\circ} \mathrm{C}$ for $5 \mathrm{sec}, 60^{\circ} \mathrm{C}$ for $45 \mathrm{sec}$ and $72^{\circ} \mathrm{C} 45 \mathrm{sec}$, repeated for 40 cycles. The primers for RT-qPCR were as follows: BDNF, forward primer 5'-AGGTCTGACGACGACATCACT-3' and reverse primer, 5'-CTTCGTTGGGCCGAACCTT-3'; VEGF, forward primer 5'-GCACATAGAGAGAATGAGCTTCC-3' and reverse primer 5'-CTCCGCTCTGAACAAGGCT-3'; miR-705, forward primer 5'-AGTAGTGGTGGGAGGTGG GGTGGGC-3' and reverse primer 5'-AGGGAGGTAGGG AGGACTGC-3'. $\beta$-actin was used as the internal control: Forward primer, 5'-CCTGTATGCCTCTGGTCG-3'; reverse primer, 5'-GGCGTAACCCTCGTAGAT-3'.

Western blotting. Brain tissues were isolated from different groups. Subsequently, $1 \mathrm{ml}$ lysis buffer was added to extract the proteins. The quantity of proteins was determined by Bicinchoninic Acid protein assay kit (Pierce Biotechnology, Inc., Rockford, IL, USA). The protein samples were boiled for $5 \mathrm{~min}$. A total of $30 \mu \mathrm{g}$ of proteins were separated on $10 \%$ 
SDS polyacrylamide gels, then transferred to polyvinylidene fluoride (PVDF) membrane (EMD Millipore, Billerica, MA, USA). The PVDF membrane was blocked with 5\% non-fat milk for $1 \mathrm{~h}$ at room temperature, and immunoblotted with antibodies against BDNF (catalog no. sc-546, 1:500) or VEGF (catalog no. sc-152, 1:500) (Santa Cruz Biotechnology, Inc., Dallas, TX, USA), respectively overnight at $4^{\circ} \mathrm{C}$. Subsequently, the membranes were washed three times with Tris-buffered saline with Tween-20 (20 mM Tris, $\mathrm{pH} 7.5 ; 150 \mathrm{mM} \mathrm{NaCl}$; $0.1 \%$ Tween-20), and incubated with the horseradish peroxidase-labeled secondary antibody (1:5,000, catalog no. LK-GAR007, MultiSciences Biotechnology Corporate Ltd., Hangzhou, Zhejiang, China) for $1 \mathrm{~h}$ at room temperature. The bands were visualized by Enhanced Chemiluminescence (Pierce; Thermo Fisher Scientific, Inc.) and analyzed by Quantity One software version 4.4 (Bio-Rad Laboratories, Inc.). $\beta$-actin (1:3,000, catalog no. A5441; Sigma-Aldrich) was used as the internal control.

Hematoxylin eosin and terminal deoxynucleotidyl transferase dUTP nick end labeling (TUNEL) staining. The brains were removed and postfixed in freshly prepared ice $4 \%$ paraformaldehyde, dehydrated with graded alcohols and embedded in paraffin. The paraffin-embedded sections with 3-4 $\mu \mathrm{m}$ were deparaffinized with xylene and rehydrated with graded alcohol, then stained with hematoxylin and eosin (HE). The images of slices at $\mathrm{x} 400$ magnification were analyzed by a light microscope. Three fields were randomly selected from the damaged area.

TUNEL staining was performed to visualize apoptotic cells according to the manufacturer's protocols (In Situ Cell Death Detection kit; POD; Roche Diagnositics, Basel, Switzerland). Briefly, subsequent to being deparaffinized, the sections were incubated with proteinase $\mathrm{K}$ and permeabilized with $0.5 \%$ Triton $\mathrm{X}-100$. Then the sections were incubated with TUNEL reaction mixture for $60 \mathrm{~min}$. For each sample, five non-overlapping fields at x400 magnification in sections were randomly captured using a fluorescence inverted microscope. The apoptotic cells were stained in a green color.

Statistical analysis. All data were expressed as the mean \pm standard deviation, and were analyzed using SPSS 20 software (IBM Corp., Armonk, NY, USA) with a one-way analysis of variance followed by the Least Significant Difference post hoc test. A value of $\mathrm{P}<0.05$ was considered to indicate a statistically significant difference. Each experiment was repeated for a minimum of three times. For RT-qPCR and western blotting experiments, each mouse sample was expressed as a ratio to its corresponding $\beta$-actin value.

\section{Results}

A lentiviral expression vector for miR-705 was successfully constructed. To examine the validity of the plasmid, the recombinant plasmid (pCDH-CMV-MCS-EF1-copG FP-miR-705) was digested with two restriction enzymes. Approximately $960 \mathrm{bp}$ of the fragment of interest was detected as presented in Fig. 1A. Combined with the DNA sequencing results, it was concluded that the overexpression vector for miR-705 was successfully constructed. To verify
Table I. Neurological deficit scores from different groups.

Neurological

Groups deficit scores

Sham group

0

Phosphate-buffered saline group

$9.34 \pm 1.21$

BMSCs-Ad

$6.32 \pm 1.33$

BMSCs-Ad-microRNA-705

$2.13 \pm 1.14$

Data are presented as the mean \pm standard deviation. BMSC, bone mesenchymal stem cell; Ad, adenovirus.

the transfection efficiency, 293T cells were transfected with pCDH-CMV-MCS-EF1-copGFP-miR-705. GFP was clearly expressed in $293 \mathrm{~T}$ cells (Fig. 1B) and BMSCs cells (Fig. 1C). These results indicated that a lentiviral expression vector for miR-705 had been successfully constructed.

BMSCs-Ad-miR-705 mitigates the neurological impairment in ischemic brain injury mice. The effect of different treatments on nerve function were evaluated by neurological deficit scores (Nds), according to a previous study (14). As presented in Table I, the sham operation has no impairment on nerve function. The NDs of PBS group were the highest, suggesting that nerve function was severely damaged. The NDs of BMSCs group was reduced, indicating that BMSCs transplantation partly reduced the neurological impairment. In addition, compared with PBS and BMSCs groups, the NDs of BMSCs-Ad-miR-705 were the lowest with a significant difference $(\mathrm{P}<0.05)$. These results indicated that BMSC transplantation mitigated the neurological impairment, and that Ad-miR-705 infection improved the repair capacity of BMSCs.

BMSCs-Ad-miR-705 suppresses neuronal cell apoptosis in $M C A O$-induced cerebral infarction. To examine the effect of BMSC transplantation on neuronal morphology, brain tissues were isolated from different groups and HE staining was conducted. In Fig. 2, normal neuronal structure and morphology were observed in the sham group. In the PBS group, MCAO resulted in marked neuronal injury with loose nerve fibers, and certain neocortical neurons were clearly damaged with characteristic vacuolation. Transplantation with BMSCs or BMSCs-Ad-miR-705 markedly improved neurological recovery, for example the number of neurons was increased and the neuronal morphology became normal. In addition, BMSCs-Ad-miR-705 enhanced the effect of BMSCs on neuronal morphology recovery.

The TUNEL assay was used to further detect the effect of BMSCs on neuronal cell apoptosis. Few apoptotic cells were identified in the sham group, and different apoptotic rates were observed in the three other groups in Fig. 3. The apoptotic rate of the PBS group was the greatest at $\sim 50 \%$. BMSC transplantation partly reduced the apoptotic rate $(27 \%)$. And the apoptotic rate of BMSCs-Ad-miR-705 was the lowest at $\sim 13 \%$. These results indicated that BMSCs-Ad-miR-705 significantly inhibited cell apoptosis caused by MCAO. 


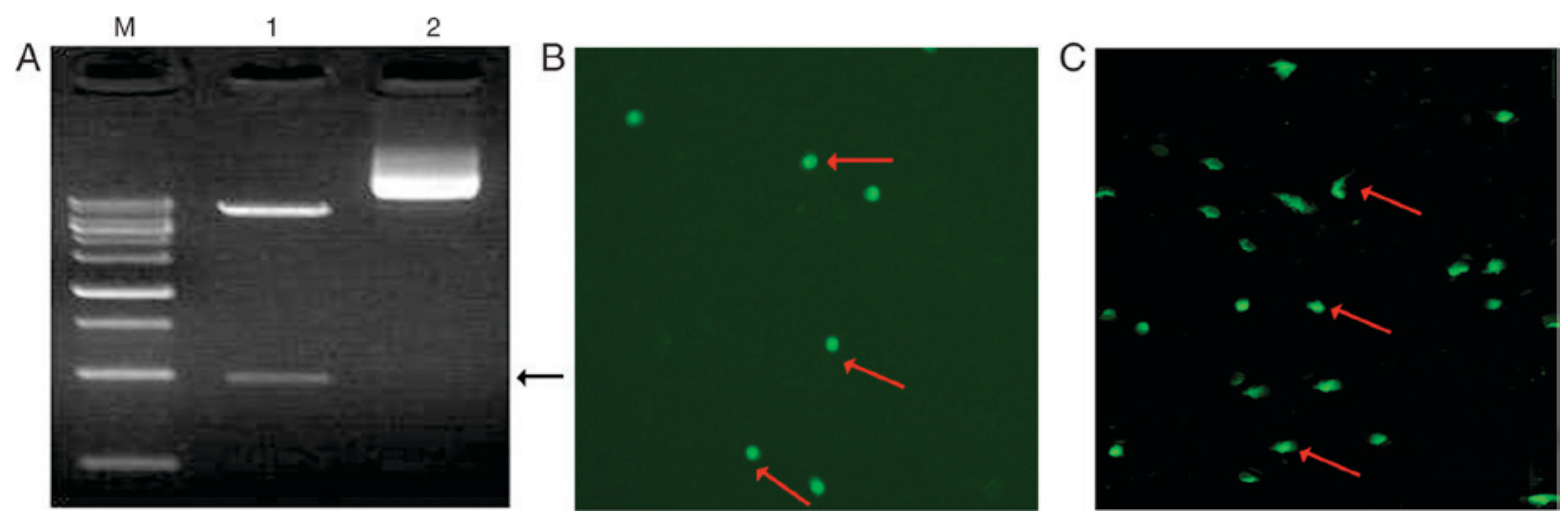

Figure 1. The lentiviral expression vector for miR-705 was successfully constructed. (A) The recombinant plasmid was verified by double enzyme digestion. The recombinant plasmid was digested by two enzymes: $X b a \mathrm{I}$ and BamHI. The target fragment was marked with a black arrow. (B) GFP was expressed in $293 \mathrm{~T}$ cells after lentiviral vector transfection, detected using a fluorescence inverted microscope (magnification, $\mathrm{x} 400$ ). (C) GFP was expressed in bone mesenchymal stem cell after lentiviral particle infection as detected by a fluorescence inverted microscope (x400). The cells with GFP expression are indicated by the red arrows. miR, microRNA; GFP, green fluorescent protein; M, marker; 1, plasmid after digestion; 2, plasmid before enzyme digestion.
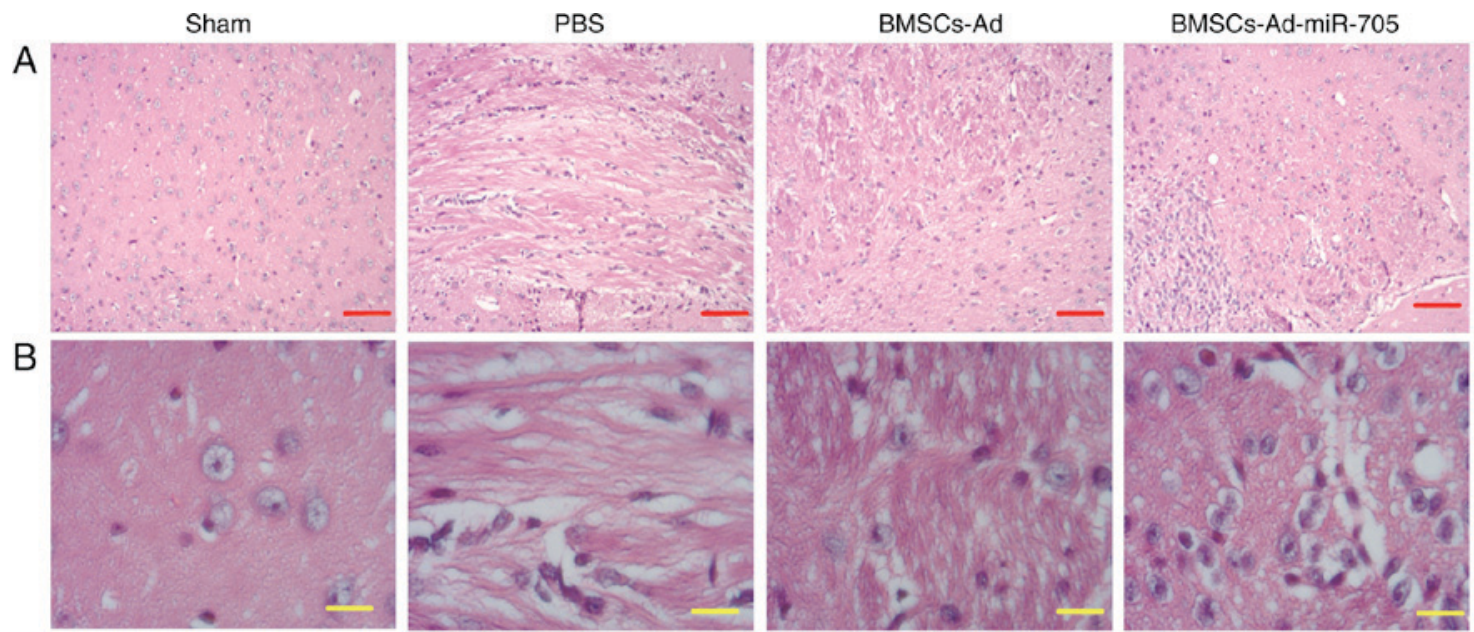

Figure 2. The morphological structure of damaged brain tissues from sham, PBS, BMSCs-Ad and BMSCs-Ad-miR-705 groups was observed by hematoxylin and eosin staining. The nuclei were stained in blue, and the cytoplasm was pink. (A) The low-power field (10x10). Scale bar in red=50 $\mu \mathrm{m}$. (B) The high-power field (40x10). Scale bar in yellow=20 $\mu \mathrm{m}$. PBS, phosphate-buffered saline; BMSC, bone mesenchymal stem cell; Ad, adenovirus; miR, microRNA.

BMSCs-Ad-miR-705 improves BDNF and VEGF expression on $m R N A$ and protein levels. To investigate the mechanism of BMSCs-Ad-miR-705 stimulating neurological recovery, the expression of two factors, BDNF and VEGF, were examined in brain tissues. Compared with the sham, PBS and BMSCs groups, the BMSCs-Ad-miR-705 group significantly increased the expression of miR-705, reconfirming the successful construction of Ad-miR-705. In Fig. 4, low and stable levels of BDNF, VEGF and miR-705 were detected in the sham group. Compared with the sham group, the expression levels of BDNF, VEGF and miR-705 were upregulated with significance $(\mathrm{P}<0.05)$. However, the levels of BDNF, VEGF and miR-705 in BMSCs-Ad and BMSCs-Ad-miR-705 groups were increased compared with those in the PBS group. The levels of BDNF, VEGF and miR-705 in BMSCs-Ad-miR-705 group were the greatest. In agreement, similar results were detected when protein expression levels of BDNF and VEGF were measured using western blotting (Fig. 5). This suggested that BMSC transplantation stimulated the expression of BDNF, VEGF and miR-705, and Ad-miR-705 infection more significantly enhanced the mRNA and protein levels of BDNF and VEGF.

\section{Discussion}

The present study demonstrated that administration of BMSCs can effectively improve functional recovery in animal models of cerebral ischemia, which is in agreement with that of a previous study (15). The current study identified that BMSCs-Ad-miR-705 significantly ameliorated neurological deficit, suppressed neuronal apoptosis and improved the expression of BDNF and VEGF in mice with ischemic brain injury. Taken together, these results indicate that injection of BMSCs-Ad-miR-705 is a potential and effective therapy for neurological recovery following cerebral ischemic injury.

In the present study, a lentiviral recombined expression vector was constructed for miR-705 overexpression. GFP was detected in plasmid-transfected 293T cells and lentiviral particle-infected BMSCs, indicating successful construction of Ad-miR-705. In Fig. 4, the highest expression level 

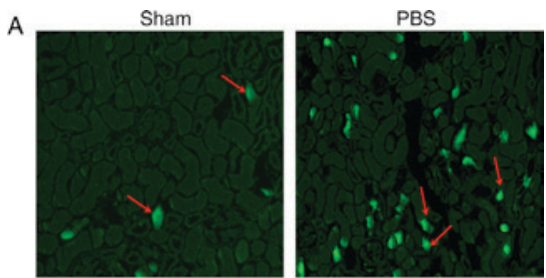

BMSCs-Ad

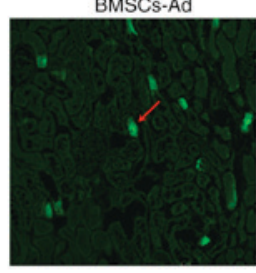

BMSCs-Ad-miR-705
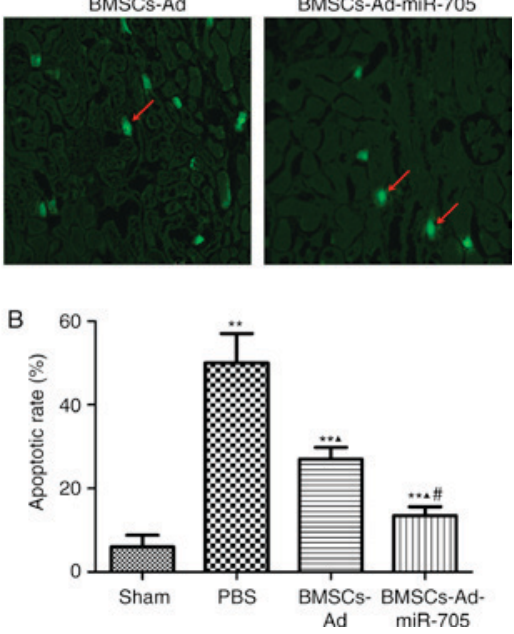

Figure 3. The cell apoptosis was detected by the TUNEL assay. (A) Images of TUNEL-positive cells were observed with a fluorescence inverted microscope $(\mathrm{x} 400)$. The damaged brain tissues of sham, PBS, BMSCs-Ad and BMSCs-Ad-miR-705 groups were extracted and cut into 3-4 $\mu \mathrm{m}$ sections for TUNEL assay. TUNEL-positive cells were green marked with red arrows. (B) The apoptotic rates of (A) were quantified. ${ }^{* *} \mathrm{P}<0.01$ vs. sham group; ${ }^{\Delta} \mathrm{P}<0.05$ vs. PBS group; ${ }^{\#} \mathrm{P}<0.05$ vs. BMSCs-Ad group. TUNEL, terminal deoxynucleotidyl transferase dUTP nick-end labeling; PBS, phosphate-buffered saline; BMSC, bone mesenchymal stem cell; Ad, adenovirus; miR, microRNA.

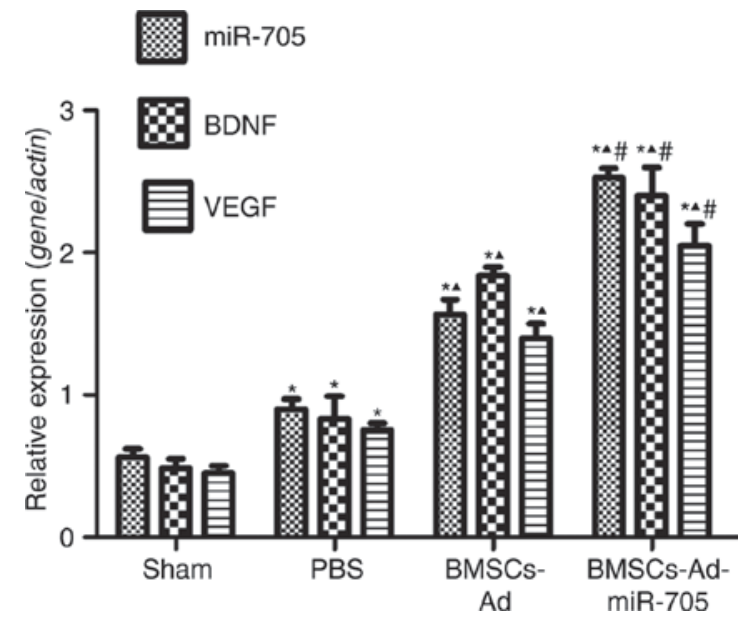

Figure 4. The mRNA levels of miR-705, BDNF and VEGF were detected by RT-qPCR. Total RNA of damaged brain tissues were isolated for the RT-qPCR assay. $\beta$-actin was used as the internal control. ${ }^{*} \mathrm{P}<0.05$ vs. sham group; ${ }^{\wedge} \mathrm{P}<0.05$ vs. PBS group; ${ }^{\#} \mathrm{P}<0.05$ vs. BMSCs-Ad group. $\mathrm{miR}$, microRNA; BDNF, bone-derived neurotrophic factor; VEGF, vascular endothelial growth factor; RT-qPCR, reverse transcription-quantitative polymerase chain reaction.

of miR-705 was observed in BMSCs-Ad-miR-705-injected mice, suggesting that miR-705 was successfully overexpressed directed by Ad-miR-705. Chen et al (16) identified that Ad-VEGF could infect BMSCs and direct the expression of

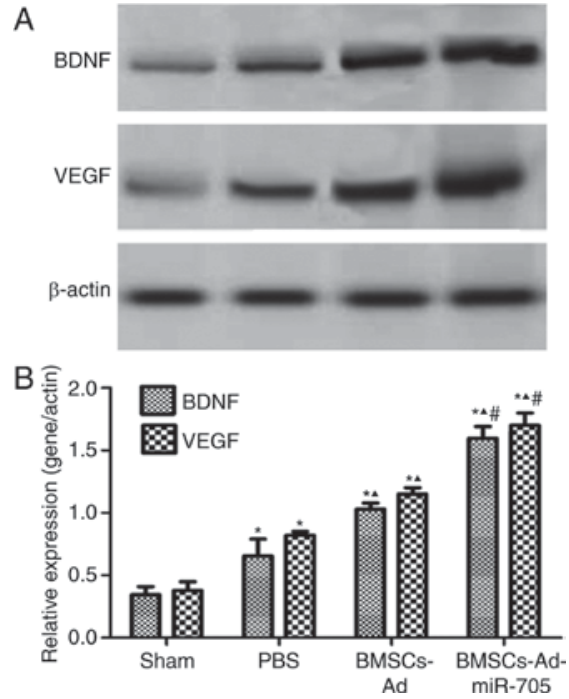

Figure 5. The protein levels of BDNF and VEGF were examined by western blotting. (A) Images of western blotting. Proteins from sham, PBS, BMSCs-Ad and BMSCs-Ad-miR-705 groups were extracted for western blotting. $\beta$-actin was used as the internal control. (B) Bands were quantified using Quantity One software. ${ }^{*} \mathrm{P}<0.05$ vs. sham group; ${ }^{\mathbf{}} \mathrm{P}<0.05$ vs. $\mathrm{PBS}$ group; ${ }^{\#} \mathrm{P}<0.05$ vs. BMSCs-Ad group. BDNF, bone-derived neurotrophic factor; VEGF, vascular endothelial growth factor; PBS, phosphate-buffered saline; BMSC, bone mesenchymal stem cell; Ad, adenovirus; miR, microRNA.

VEGF in BMSCs. Combined with the results of the present study, it is suggested that this method of lentivirus-mediated overexpression of target genes in BMSCs provides novel insight for cellular therapy.

miR-705 was first identified to be significantly expressed in BMSCs from osteoporosis bone marrow. TNF $\alpha$ and ROS have been observed to mediate the overexpression of miR-705 to regulate the cell lineage commitment of BMSCs through NF- $\kappa \mathrm{B}$ signaling (12). The present study focused on investigating the function of miR-705 in cerebral ischemic damage. In mice with cerebral ischemia, intravenous BMSC therapy reduced cell apoptosis and promoted endogenous cell proliferation (17), and stimulated functional recovery for brain repair (18). In the current study, compared with the PBS group, the NDs of the BMSCs-Ad group were decreased, the neurological morphology was improved and the apoptosis rate of neurons were declined. These results were in agreement with the abovementioned previous research. At present, two targets of miR-705 have been reported: HOXA10 and FoxO1. HOXA10 is a member of the family of homeodomain-containing transcription factors, and its overexpression inhibits adipocyte differentiation of BMSCs (12). However, the decrease of FoxO1 leads to oxidative damage and inhibition of BMSC differentiation (13). According to the results of the current study, it was suggested that HOXA10 is the main target gene of miR-705 in improving BMSC therapy. However, further experiments are required.

Previous studies have indicated that administration of BMSCs facilitates the release of neurovascular trophic factors from activated astrocytes, including BDNF, nerve growth factor, VEGF and basic fibroblast growth factor through activating astrocytic phosphatidylinositol 3-kinase (PI3K) and extracellular signal-regulated kinase pathways $(19,20)$. During the process of neurological recovery, these factors reduced 
neuronal apoptosis and enhanced neuronal regeneration and cellular differentiation (21). It was observed that BMSC transplantation increased the expression of BDNF and VEGF. Mature BDNF is crucial in the protection of the neonatal or developing brain from ischemia injury (5). Administration of BMSCs-BDNF into mice significantly promoted functional recovery and reduced the numbers of TUNEL-positive apoptotic cells (22). VEGF promotes the formation of new cerebral blood vessels, and injection of VEGF improves neurological function and protects the brain against ischemia (23). VEGF-BMSCs combination therapy ameliorates ischemic damage by the activation of the PI3K/protein kinase $\mathrm{B} /$ glycogen synthase kinase $3 \beta$ signaling pathway (16). VEGF, in addition to BDNF, has been implicated in the regulation of brain neurogenesis by promoting the proliferation and differentiation of neuronal precursors (24). The current on the effect of BMSCs on the expression of BDNF and VEGF was in agreement with the previous data and it was observed that BMSCs-Ad-miR-705 enhanced the outcome of BMSCs transplantation on NDs improvement, neuronal apoptosis and the expression VEGF and BDNF. Thus, these results provided novel insight into that the role of miR-705 in function neuronal recovery via induction of the secretion of growth factors, and indicated miR-705 as a potential therapeutic target for stem cell-mediated regenerative medicine for cerebral ischemia.

In summary, BMSCs-Ad-miR-705 promoted the secretion of VEGF and BDNF, suppressed the neuronal apoptosis and stimulated the neuronal regeneration, in turn functioning in the impairment of ischemic brain damage. However, the mechanism by which miR-705 functioned in the BMSCs-mediated signaling pathway requires further investigation.

\section{References}

1. Wang Hy, Wang Gl, Yu Yh and Wang Y: The role of phosphoinositide-3-kinase/Akt pathway in propofol-induced postconditioning against focal cerebral ischemia-reperfusion injury in rats. Brain Res 1297: 177-184, 2009.

2. Schouten JW, Fulp CT, Royo NC, Saatman KE, Watson DJ, Snyder EY, Trojanowski JQ, Prockop DJ, Maas AI and McIntosh TK: A review and rationale for the use of cellular transplantation as a therapeutic strategy for traumatic brain injury. J Neurotrauma 21: 1501-1538, 2004

3. Yang Z, Cai X, Xu A, Xu F and Liang Q: Bone marrow stromal cell transplantation through tail vein injection promotes angiogenesis and vascular endothelial growth factor expression in cerebral infarct area in rats. Cytotherapy 17: 1200-1212, 2015.

4. Shichinohe H, Ishihara T, Takahashi K, Tanaka Y, Miyamoto M, Yamauchi T, Saito H, Takemoto H, Houkin K and Kuroda S: Bone marrow stromal cells rescue ischemic brain by trophic effects and phenotypic change toward neural cells. Neurorehab Neural Repair 29: 80-89, 2015.

5. Chen A, Xiong LJ, Tong Y and Mao M: The neuroprotective roles of BDNF in hypoxic ischemic brain injury (Review). Biomed Rep 1: 167-176, 2013.

6. Sun Y, Jin K, Xie L, Childs J, Mao XO, Logvinova A and Greenberg DA: VEGF-induced neuroprotection, neurogenesis, and angiogenesis after focal cerebral ischemia. J Clin Invest 111: 1843-1851, 2003

7. Bartel DP: MicroRNAs: Target recognition and regulatory functions. Cell 136: 215-233, 2009.
8. Yoshino H, Seki N, Itesako T, Chiyomaru T, Nakagawa M and Enokida H: Aberrant expression of microRNAs in bladder cancer. Nat Rev Urol 10: 396-404, 2013.

9. Bartel DP: MicroRNAs: Genomics, biogenesis, mechanism, and function. Cell 116: 281-297, 2004.

10. Lian JB, Stein GS, Van Wijnen AJ, Stein JL, Hassan MQ, Gaur T and Zhang Y: MicroRNA control of bone formation and homeostasis. Nat Rev Endocrinol 8: 212-227, 2012.

11. Dolganiuc A, Petrasek J, Kodys K, Catalano D, Mandrekar P, Velayudham A and Szabo G: MicroRNA expression profile in Lieber-DeCarli diet-induced alcoholic and methionine choline deficient diet-induced nonalcoholic steatohepatitis models in mice. Alcohol Clin Exp Res 33: 1704-1710, 2009.

12. Liao L, Yang X, Su X, Hu C, Zhu X, Yang N, Chen X, Shi S, Shi S and Jin Y: Redundant miR-3077-5p and miR-705 mediate the shift of mesenchymal stem cell lineage commitment to adipocyte in osteoporosis bone marrow. Cell Death Dis 4: e600, 2013.

13. Liao L, Su X, Yang X, Hu C, Li B, Lv Y, Shuai Y, Jing H, Deng Z and Jin Y: TNF- $\alpha$ Inhibits FoxO1 by Upregulating miR-705 to aggravate oxidative damage in bone marrow-derived mesenchymal stem cells during osteoporosis. Stem Cells 34: 1054-1067, 2016.

14. Schallert T, Kozlowski DA, Humm JL and Cocke RR: Use-dependent structural events in recovery of function. Adv Neurol 73: 229-238, 1997.

15. Pirzad Jahromi G, Seidi S, Sadr SS, Shabanzadeh AP, Keshavarz M, Kaka GR, Hosseini SK, Sohanaki H and Charish J: Therapeutic effects of a combinatorial treatment of simvastatin and bone marrow stromal cells on experimental embolic stroke. Basic Clin Pharmacol Toxicol 110: 487-493, 2012.

16. Chen B, Zhang F, Li QY, Gong A and Lan Q: Protective effect of Ad-VEGF-bone mesenchymal stem cells on cerebral infarction. Turk Neurosurg 26: 8-15, 2016.

17. Gutiérrez-Fernández M, Rodríguez-Frutos B, Ramos-Cejudo J, Teresa Vallejo-Cremades M, Fuentes B, Cerdán S and Díez-Tejedor E: Effects of intravenous administration of allogenic bone marrow-and adipose tissue-derived mesenchymal stem cells on functional recovery and brain repair markers in experimental ischemic stroke. Stem Cell Res Ther 4: 11, 2013.

18. Wang L, Lin Z, Shao B, Zhuge Q and Jin K: Therapeutic applications of bone marrow-derived stem cells in ischemic stroke. Neurol Res 35: 470-478, 2013.

19. Wu J, Sun Z, Sun HS, Wu J, Weisel RD, Keating A, Li ZH, Feng ZP and Li RK: Intravenously administered bone marrow cells migrate to damaged brain tissue and improve neural function in ischemic rats. Cell Transplant 16: 993-1005, 2008.

20. Zhang Q, Chen ZW, Zhao YH, Liu BW, Liu NW, Ke CC and Tan HM: Bone marrow stromal cells combined with sodium ferulate and n-butylidenephthalide promote the effect of therapeutic angiogenesis via advancing astrocyte-derived trophic factors after ischemic stroke. Cell Transplant 26: 229-242, 2017.

21. Wan H, Li F, Zhu L, Wang J, Yang Z and Pan Y: Update on therapeutic mechanism for bone marrow stromal cells in ischemic stroke. J Mol Neurosci 52: 177-185, 2014.

22. Yao RQ, Qi DS, Yu HL, Liu J, Yang LH and Wu XX: Quercetin attenuates cell apoptosis in focal cerebral ischemia rat brain via activation of BDNF-TrkB-PI3K/Akt signaling pathway. Neurochem Res 37: 2777-2786, 2012.

23. Li N, Wang P, Ma XL, Wang J, Zhao LJ, Du L, Wang LY, Wang XR and Liu KD: Effect of bone marrow stromal cell transplantation on neurologic function and expression of VEGF in rats with focal cerebral ischemia. Mol Med Rep 10: 2299-2305, 2014.

24. Deindl E: Mechanistic insights into the functional role of vascular endothelial growth factor and its signalling partner brain-derived neurotrophic factor in angiogenic tube formation. Acta Physiol (Oxf) 211: 268-270, 2014. 